\title{
Diagnostics for African trypanosomiasis and prospects of improved staging of the disease Joseph Ndung'u
}

\author{
Address: Foundation for Innovative New Diagnostics (FIND), 71 Avenue Louis Casai, P.O. Box 93, 1216 Cointrin, Switzerland
}

Email: Joseph Ndung'u - joseph.ndungu@finddiagnostics.org

from Infectious diseases of the nervous system: pathogenesis and worldwide impact

Paris, France. 10-13 September 2008

Published: 23 September 2008

BMC Proceedings 2008, 2(Suppl I):S33

This abstract is available from: http://www.biomedcentral.com/I753-656I/2/SI/S33

(c) 2008 Ndung'u; licensee BioMed Central Ltd.

Control of Human African trypanosomiasis (HAT) relies on diagnosis and treatment of infected individuals, and vector control. None of the diagnostic tests for HAT are produced commercially, and the available ones suffer from inadequate sensitivity and specificity. There is little interest to invest in diagnostics for neglected diseases such as HAT, a disease of poverty that is confined to specific foci in rural Africa. The Foundation for Innovative New Diagnostics (FIND) is working with partners to develop new tools for HAT. Two types of tests are being developed: 1) a test for infection with Trypanosoma brucei gambiense and/or T.b. rhodesiense and 2) a staging test to determine whether the central nervous system (CNS) is affected, and to guide treatment and follow-up. Staging HAT is essential as the drugs that treat patients with brain involvement are quite toxic. Staging involves a lumbar puncture, which is invasive and painful. The cut-off criteria for staging have an intermediate area of uncertainty, and treated patients have to be followed for 24 months to confirm cure.

A number of feasibility projects are going on, focusing on parasite separation from blood and CSF, serological and molecular tests, and staging of the disease. The miniature anion exchange centrifugation technique (mAECT) kit for separation of parasites has been improved and its manufacture re-established in a disease endemic country. Development of a robust and affordable Primostar iLED fluorescence microscope that is ideal for African settings has improved prospects for better diagnosis of HAT. The microscope is being evaluated for viewing trypanosomes stained with Acridine Orange, and later with parasite-spe- cific markers. The markers include labeled single-chain variable fragment antibodies, aptamers and nanobodies, which are also undergoing feasibility testing in detecting trypanosome antigens. Screening of recombinant and synthetic antigens for the development of an antibody detection test will result in selection of the best to be used in developing the test. The feasibility of using the loopmediated isothermal amplification (LAMP) of DNA in diagnosis of HAT has been proven, and development into a kit is to start soon.

New methods for staging and follow-up are being explored, with very encouraging results. These range from improvement of existing tests, marker validation, to discovery research. Special attention is being given to speed, simplicity, cost and reliability of the tests, as well as reduced invasiveness. 